\title{
Comparison of porcine milk microRNA expression in milk exosomes versus whole swine milk and prediction of target genes
}

\author{
Zhihong Liu ${ }^{1,2,3,4, \star}$, Yuchun Xie ${ }^{1,2,3,4, \star}$, Juntao Guo ${ }^{1,2,3,4}$, Xin Su$^{1,2,3,4}$, Cun Zhao ${ }^{1,2,3,4}$, \\ Chongyan Zhang ${ }^{1,2,3,4}$, Qing Qin ${ }^{1,2,3,4}$, Dongliang Dai ${ }^{1,2,3,4}$, Yanan Tuo ${ }^{1,2,3,4}$, Zongyuan $\mathbf{L i}^{1,2,3,4}$, \\ Danni $\mathbf{W u}^{1,2,3,4}$, and Jinquan $\mathbf{L i}^{1,2,3,4}$ \\ ${ }^{1}$ College of Animal Science, Inner Mongolia Agricultural University, Hohhot 010018, Inner Mongolia, China \\ ${ }^{2}$ Key Laboratory of Animal Genetics, Breeding and Reproduction, Hohhot 010018, \\ Inner Mongolia Autonomous Region, China \\ ${ }^{3}$ Key Laboratory of Mutton Sheep Genetics and Breeding, Ministry of Agriculture, Hohhot 010018, China \\ ${ }^{4}$ Engineering Research Center for Goat Genetics and Breeding, Hohhot 010018, \\ Inner Mongolia Autonomous Region, China \\ « These authors contributed equally to this work.
}

Correspondence: Jinquan Li (lijinquan_nd@126.com) and Zhihong Liu (liuzh7799@163.com)

Received: 12 February 2021 - Revised: 5 September 2021 - Accepted: 29 November 2021 - Published: 26 January 2022

\begin{abstract}
Milk exosomal microRNAs (miRNAs) are important for postnatal growth and immune system maturation in newborn mammals. The functional hypothesis of milk exosomal miRNAs and their potential bioavailability in milk to newborn mammals were investigated. Briefly, 37 exosomal miRNAs were upregulated compared to miRNAs found outside the exosomes. Among these miRNAs, ssc-miR-193a-3p expression was upregulated 1467.35 times, while ssc-miR-423-5p, ssc-miR-551a, ssc-miR-138, ssc-miR-1 and ssc-miR-124a were highly concentrated and upregulated 13.58-30.06 times. Moreover, these miRNAs appeared to be relevant for cell development and basic physiological processes of the immune system. Following the analysis of target gene prediction and related signalling pathways, 9262 target genes were mainly concentrated in three signalling pathways: metabolic pathways, pathways in cancer, and phosphatidylinositol 3-kinase/protein kinase B (PI3K/Akt) signalling pathways. Among 9262 target genes, more than 20 miRNAs were enriched in exosomes, such as methyl CpG binding protein 2 (MECP2) and glycogen synthase 1 (GYS1). After determining the miRNA localization-, distribution- and function-related metabolism, we found that these exosomes were specifically concentrated miRNA target genes and they were interrelated with cell development and basic cell functions, such as metabolism and immunity. It is speculated that miRNAs in milk can influence offspring via milk exosomes.
\end{abstract}

\section{Introduction}

Milk is an important source of nutrition and a vehicle of passive immunity for infants. It contains numerous immune components indispensable for infant brain development and growth. Additionally, it is a rich source of miRNAs that are essential for neonatal innate and adaptive immunity. There are mutually exclusive hypotheses regarding the function of mother's milk: (1) that the miRNAs in milk can be passed to the newborn and thus are relevant for physiologic adjustment and (2) that milk merely provides nutritional benefits (Melnik et al., 2016).

Chen et al. (2010) detected 245 different kinds of miRNAs in cow's milk. Moreover, using Illumina's Solexa Sequencing Technology, it has been confirmed that 213 different kinds of miRNAs are present in ordinary cow milk (Chen et al., 2010). RNAs of bovine milk exosomes have been detected in tissues (including the brain) of mice after oral ad- 
ministration of milk exosomes (Manca et al., 2018). Furthermore, miRNAs in animal body fluids have often been shown to be selectively wrapped in exosomes. Kosaka et al. (2010) found that miRNA molecules are stable even in very acidic conditions, indicating that milk allows for the dietary intake of miRNAs by infants. miRNAs present in milk were able to enter normal and tumour cells and affect their biological functions. For example, milk exosome-derived miRNA-148a via DNMT1 suppression reduces the methylation status of $\mathrm{CpG}$ islands of promoters of crucial developmental genes (Golan-Gerstl et al., 2017).

Exosomes contain a number of proteins and fats, as well as a number of nucleotides (mRNA and microRNA) (Liu et al., 2018). Exosomes have an important role in transporting RNAs to target cells. RNAs in high-content exosomes are relevant for the delivery of genetic information between tissues and low-content exosomes, e.g. tumour and immune cellderived exosomes, have been shown to carry tumour antigens and promote immunity, leading to the eradication of established tumours by $\mathrm{CD} 8+\mathrm{T}$ cells and $\mathrm{CD} 4+\mathrm{T}$ cells, as well as directly suppressing tumour growth and improving resistance to malignant tumour development (David et al., 2015). Furthermore, miRNAs in exosomes can resist RNase degradation and be preserved in serum and blood plasma, and they can be used as tags for clinical and biological diagnosis (Lin et al., 2016). Zhang et al. (2014) proposed that exosomes and their products could serve as valid gene and drug delivery carriers in cancer therapy. For example, higher miR-92a$3 p$ and miR-17-5p expression in circulating exosomes was clearly associated with the pathological stages and grades of colorectal cancer (Fu et al., 2017). miRNAs in exosomes may have an important role as delivery carriers and biological tags. We further analysed and discussed the content and function of miRNAs in swine milk exosomes.

\section{Materials and methods}

\subsection{Ethics statement}

All methods used in this study were performed in accordance with protocols approved by the Laboratory Animal Management Committee of the Chongqing Academy of Animal Sciences and the Ministry of Science and Technology of the Peoples Republic of China (approval number: 2006398).

\subsection{Animals}

Fresh swine milk samples were collected from the pig breeding farm of the Chongqing Academy of Animal Science, China. Three individuals were randomly selected for biological analysis replicates. After $31 \mathrm{~d}$ of lactation, we removed the piglets and collected swine milk, which was quickly frozen in liquid nitrogen and stored for analysis.

\subsection{Exosomal extraction}

Exosomes were isolated with the ExoQuick-TC (System Biosciences, USA) according to the manufacturer's instructions. Briefly, biofluids were collected from $10 \mathrm{~mL}$ milk and centrifuged at $3000 \times g$ for 15 min to remove cells and cell debris. Two millilitres (mL) of ExoQuick-TC Exosome Precipitation Solution was added to the supernatants, mixed by vortexing and stored at $4{ }^{\circ} \mathrm{C}$ overnight. Then, the mixture was centrifuged at $1500 \times g$ for $30 \mathrm{~min}$ at room temperature, the supernatant was discarded, and the pellet was resuspended in $100-500 \mu \mathrm{L}$ of 1X RIPA buffer. Finally, the exosomes were extracted and observed by scanning electron microscopy.

\subsection{RNA extraction}

RNA extraction was conducted as follows: $10 \mathrm{~mL}$ of sample was mixed with $2 \mathrm{~mL}$ ExoQuick-TC, placed at $4{ }^{\circ} \mathrm{C}$ overnight $(6 \mathrm{~h})$, and centrifuged at $1500 \times g$ for $30 \mathrm{~min}$, and the supernatant was discarded. The exosome pellet was resuspended in $350 \mu \mathrm{L}$ LYSIS buffer, vortexed for $15 \mathrm{~s}$, and placed at room temperature for $5 \mathrm{~min}$. The solution was then mixed with $200 \mu \mathrm{L}$ of $100 \%$ ethanol, vortexed for an additional $10 \mathrm{~s}$, transferred to a fresh spin column, and centrifuged at $13000 \mathrm{rpm}$ for $1 \mathrm{~min}$. The flow-through was discarded, and the spin column was placed back into a collection tube, mixed with $400 \mu \mathrm{L}$ of WASH buffer and centrifuged at $13000 \mathrm{rpm}$ for $1 \mathrm{~min}$. The flow-through was discarded, and the solution was centrifuged at $13000 \mathrm{rpm}$ for $2 \mathrm{~min}$ to dry. Subsequently, the collection tube was discarded, while the spin column was assembled with a fresh, RNase-free $1.5 \mathrm{~mL}$ elution tube. ELUTION buffer $(30 \mu \mathrm{L})$ was directly added to the membrane in a spin column and centrifuged at $2000 \mathrm{rpm}$ for $2 \mathrm{~min}$. The speed was increased to $13000 \mathrm{rpm}$ and it was centrifuged for $1 \mathrm{~min}$.

To control the quality of miRNA and cDNA synthesis, a miRCURY LNA ${ }^{\mathrm{TM}}$ Universal RT microRNA PCR RNA Spike-in kit (Exiqon) was used. The total RNA quantity and purity were analysed by a Bioanalyser 2100 and RNA 6000 Nano Lab Chip Kit (Agilent, CA, USA) with RIN number > 7.0. To control the quality of the RNA extraction and cDNA synthesis for microRNA qPCR, a miRCURY LNA ${ }^{\mathrm{TM}}$ Universal RT microRNA PCR RNA Spike-in kit (Exiqon) was used.

\subsection{Small RNA library construction and sequencing}

Approximately $1 \mu \mathrm{g}$ of total RNA was used to prepare a small RNA library according to the protocol of TruSeq Small RNA Sample Prep Kits (Illumina, San Diego, USA). We performed single-end sequencing ( $36 \mathrm{bp}$ ) on an Illumina HiSeq 2500 at LC-BIO (Hangzhou, China) following the vendor's recommended protocol.

The original data were initially removed from the $3^{\prime}$ adapter sequence, while sequences shorter than $18 \mathrm{nt}$ in length were discarded. If there were $80 \% \mathrm{~A}, \mathrm{G}, \mathrm{C}$, or T in 


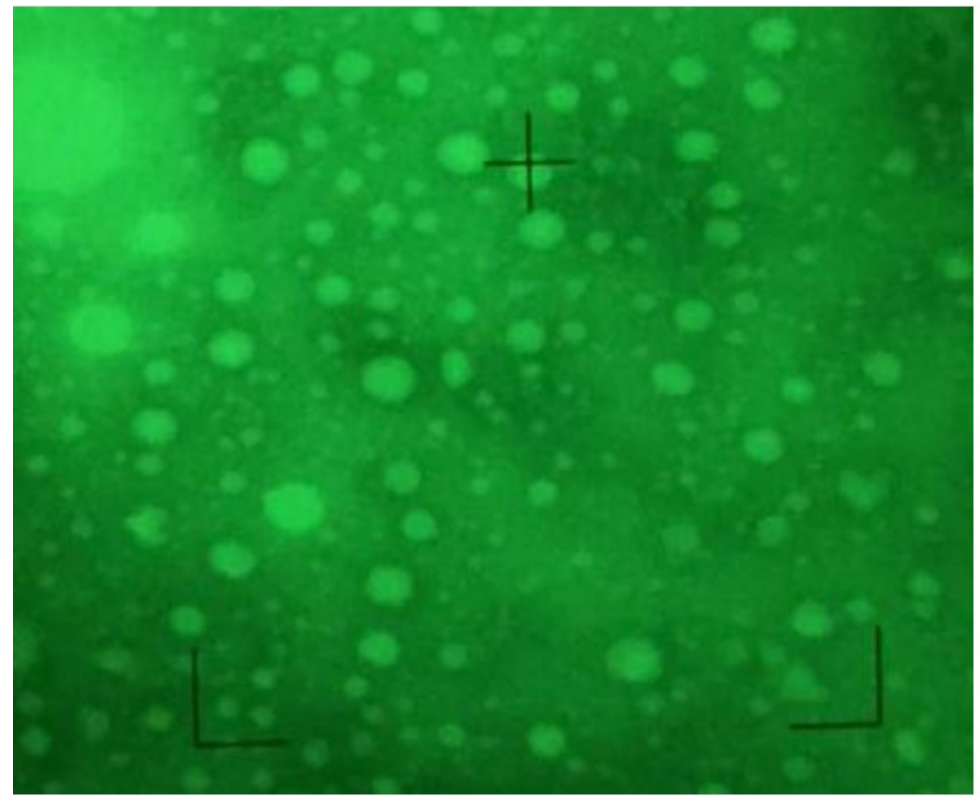

(a)

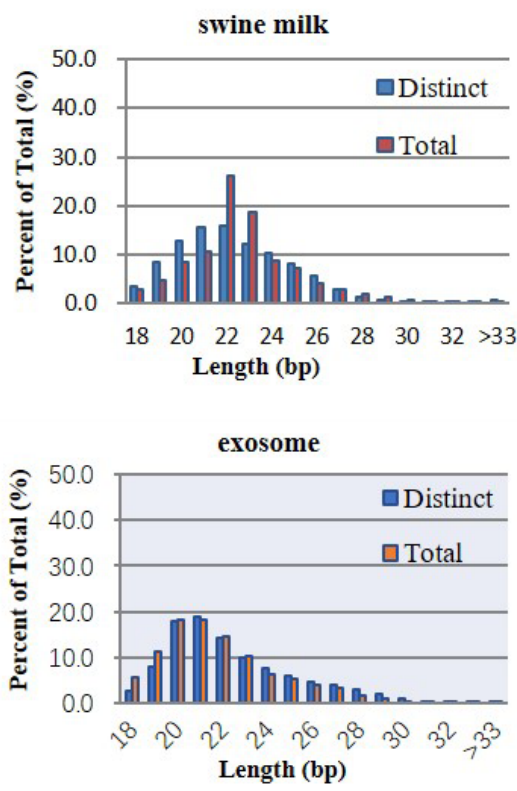

(b)

Figure 1. Basic analysis. (a) Exosomes isolated from swine milk. (b) Statistics of miRNA length from different samples.

the sequence, only $\mathrm{A}$ or $\mathrm{C}$ with no $\mathrm{G}$ or $\mathrm{T}$, or only $\mathrm{G}$ or $\mathrm{T}$ with no $\mathrm{A}, \mathrm{C}$, or continuous nucleotide dimers or trimers, these sequences were filtered out. By comparing the measured sequences with the mRNA, rRNA, tRNA, snRNA, and Repbase databases, clean reads were obtained.

\subsection{Conservative analysis of miRNA}

The remaining reads of ncRNAs such as rRNA and tRNA were removed (miRbase 20) to identify and obtain miRNA expression levels: if the mature miRNA was in the $[x, y]$ region of the pre-miRNA and the sequencing quantity was in the position $[x-2, y+5]$, it was counted as the expression amount of the mature miRNA.

\section{7 miRNA difference between exosome and swine milk}

EdgeR was used to analyse the differences between the miRNAs: a $P$ value $<0.05$ was considered statistically significant, a ratio $>2$ times higher was considered different, and a ratio $>10$ times higher was considered significantly different.

\subsection{Upregulated miRNA in exosome}

Family analysis and gene cluster analysis. According to the position of pre-miRNA in the genome, we treated the miRNA within the range $(10 \mathrm{~kb})$ as a miRNA cluster. miRNAs were classified by a family of 2 to $8 \mathrm{bp}$ seed regions of the mature body. The family name corresponding to the seed sequence was classified as a reference to Tar-
getScan (http://www.targetscan.org/, last access: 25 December 2014). Prediction of miRNA target genes with miRNAs was performed using the default parameter of free energy $<-20 \mathrm{kcal} \mathrm{mol}^{-1}$. Thirty-seven upregulated miRNAs in exosomes were analysed according to http://www.kegg.jp/ kegg/pathway.html (last access: 27 December 2014) to map pathways.

\section{Results}

\subsection{Ultrastructural features of porcine milk exosomes}

Electron microscopy revealed exosomes that are represented by small vesicles of different sizes (diameter of 40-100 nm) (Fig. 1a). The samples were of good shape, high density, low impurity, and the expected size.

\subsection{Subtractive libraries}

By sequencing swine milk samples (R-1, R-2, R-3) and exosome samples (E-1, E-2, E-3), we obtained 10088 489, 10955079,9907 545, 10785 934, 14208994 , and 16651629 clean reads. microRNAs with 18-34 bases were found in milk and exosomes, and their length was consistent with a normal distribution (approximately $22 \mathrm{nt}$ ) (Fig. 1b).

\subsection{Conservative analysis of microRNA}

By searching the swine miRNA data in miRbase 20.0 and performing bow-tie comparison, we obtained 280 pre- 


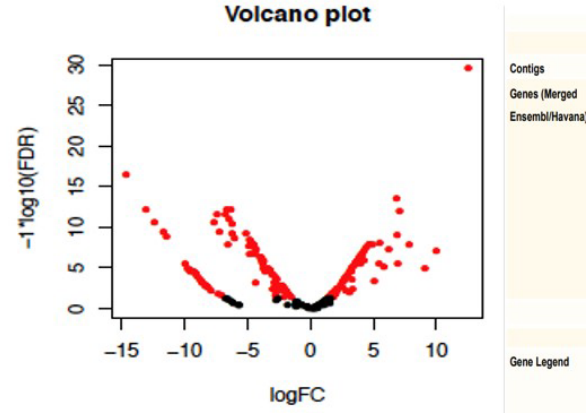

(a)

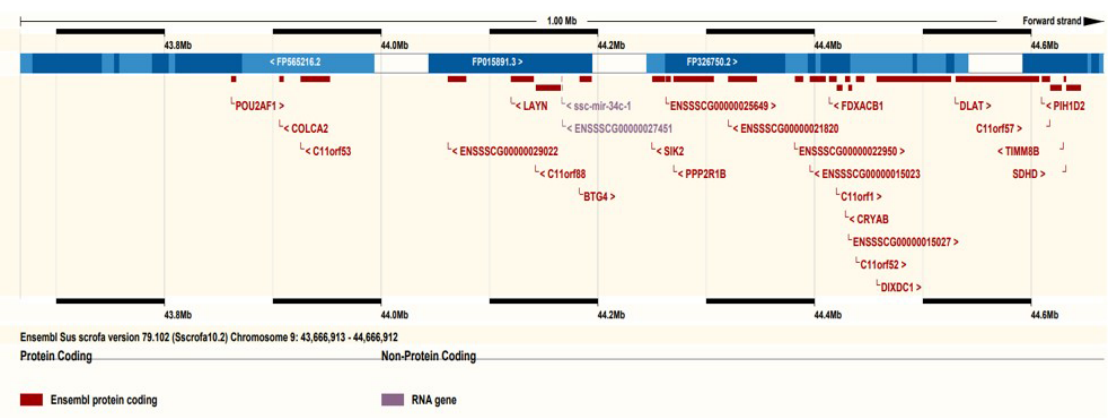

(b)

Figure 2. Bioinformatics analysis of microRNA. (a) Volcano plot of differential miRNAs between exosomes and milk (red indicates differentially expressed miRNAs). (b) Mapping of the miRNA cluster on chromosome 9.

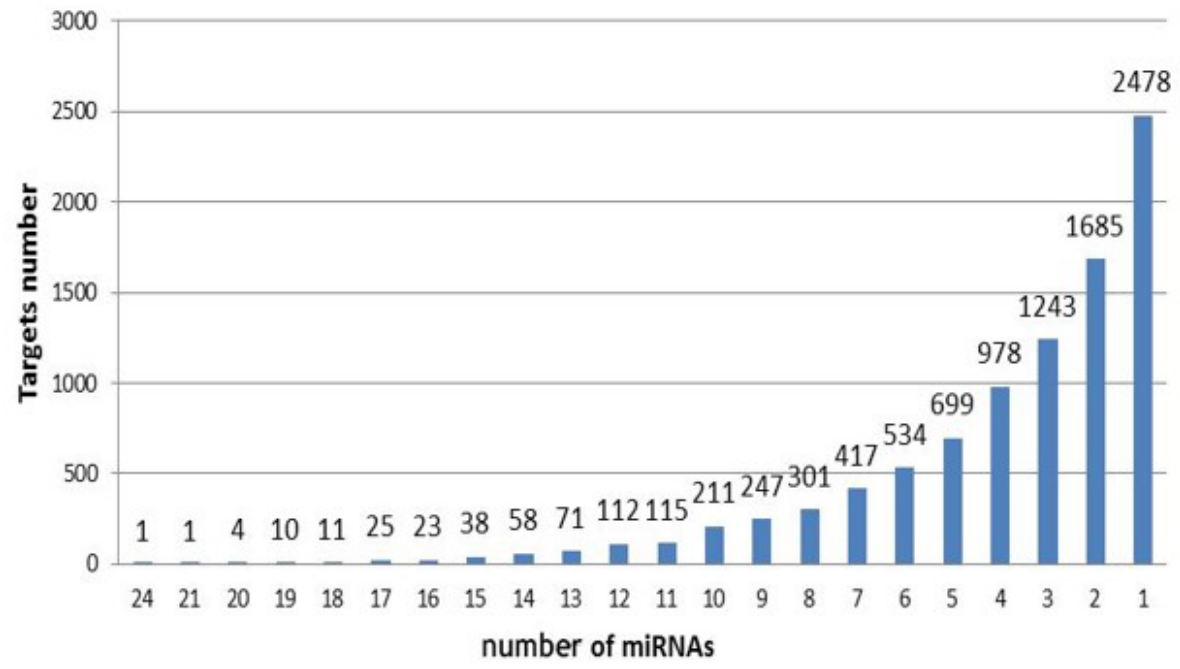

Figure 3. The number of target genes corresponding to miRNA.

miRNAs and 326 mature miRNAs (Table 1). Furthermore, 236 pre-miRNAs and 243 conserved miRNAs were found in swine milk, and 208 pre-miRNAs and 200 mature miRNAs were found in exosomes.

\section{4 miRNA content of swine milk and swine exosomes}

Compared to miRNAs found in swine milk, 2-fold higher expression was observed for 37 exosomal miRNAs and 10-fold higher expression was observed for six exosomal miRNAs (Table 2, Fig. 2a). Among these, the level of ssc-miR-193a$3 p$ expression was 1467.35 times higher in exosomes than in milk. Five additional exosomal miRNAs, i.e. ssc-miR-4235p, ssc-miR-551a, ssc-miR-138, ssc-miR-1, and ssc-miR$124 \mathrm{a}$, were found to be concentrated and upregulated (13.58 to 30.06-fold higher expression) in exosomes.

\subsection{Analysing 37 upregulated miRNAs detected in swine milk and exosomes}

Family analysis and gene cluster analysis. There were 41 pre-miRNAs obtained belonging to 30 families (Table 3). Two mature miRNAs were found in miR-146, miR-17, miR221 , and miR-30, while all of the others had a single mature miRNA.

By chromosome localization of 41 upregulated premiRNAs in exosomes and $5 \mathrm{M}$ selection, $9 \mathrm{ch}$ and $\mathrm{Xch}$ had gene clusters (Table 4). ssc-mir-34c-1 and ssc-mir-34c-2 were located in 44166873 44166952 on 9ch and fixed on 45274873_45274942 and 45275613_45275692 on Xch. Two miRNAs on 9ch are members of the miR-34 family, and the miRNAs are located on many upstream genes between the LAYN and SIK2 genes. In addition, there were new miRNAs at this site (Fig. 2b). Two miRNAs on Xch are members of the mir-221 family. There are new miRNAs in the $10 \mathrm{k}$ range where no gene exists. 
Table 1. Statistical analysis of microRNAs.

\begin{tabular}{lcccr}
\hline Class & Pre-miRNA & Mature & $\begin{array}{r}\text { Unique sRNA mapped } \\
\text { pre-miRNA }\end{array}$ & $\begin{array}{r}\text { Total sRNA mapped } \\
\text { pre-miRNA }\end{array}$ \\
\hline $\begin{array}{l}\text { Known microRNAs } \\
\text { in miRbase }\end{array}$ & 280 & 326 & - & - \\
\hline Swine milk & 236 & 243 & 2838 & 1794033 \\
\hline Exosome & 208 & 200 & 1613 & 234133 \\
\hline
\end{tabular}

Table 2. High concentration of miRNA in exosomes.

\begin{tabular}{|c|c|c|c|c|c|c|c|c|}
\hline \multirow[b]{2}{*}{ micoRNA } & \multicolumn{4}{|c|}{ Database } & \multicolumn{4}{|c|}{ Exosome vs. swine milk } \\
\hline & Sequence & Length & Swine milk & Exosome & $P$ value & Ratio & $\begin{array}{l}\text { Mark } \\
2 \text { times }\end{array}$ & $\begin{array}{l}\text { Mark } \\
10 \text { times }\end{array}$ \\
\hline ssc-miR-193a-3p & $\begin{array}{l}\text { AACTGGCCTACAAA } \\
\text { GTCCCAGT }\end{array}$ & 22 & 75 & 67154 & $1.33 \times 10^{-32}$ & 1467.35 & UP & UP \\
\hline ssc-miR-423-5p & $\begin{array}{l}\text { TGAGGGGCAGAG } \\
\text { AGCGAGACTTT }\end{array}$ & 23 & 1302 & 22588 & $4.23 \times 10^{-16}$ & 30.06 & UP & UP \\
\hline ssc-miR-551a & $\begin{array}{l}\text { GCGACCCACTCTTG } \\
\text { GTTTCC }\end{array}$ & 20 & 11 & 225 & $3.75 \times 10^{-14}$ & 25.43 & UP & UP \\
\hline ssc-miR-138 & $\begin{array}{l}\text { AGCTGGTGTTGTGA } \\
\text { ATCAGGC }\end{array}$ & 21 & 1 & 42 & $1.90 \times 10^{-9}$ & 13.95 & UP & UP \\
\hline ssc-miR-1 & $\begin{array}{l}\text { TGGAATGTAAAGAA } \\
\text { GTATGTA }\end{array}$ & 21 & 3 & 58 & $8.12 \times 10^{-11}$ & 13.94 & UP & UP \\
\hline ssc-miR-124a & $\begin{array}{l}\text { TAAGGCACGCGGTG } \\
\text { AATGCCA }\end{array}$ & 21 & 0 & 33 & $1.83 \times 10^{-8}$ & 13.58 & UP & UP \\
\hline
\end{tabular}

Target gene analysis. With target gene prediction of 37 upregulated miRNAs in exosomes, we obtained 9262 target genes and their following miRNAs (Fig. 3). Six target genes corresponded to 20 miRNAs, 674 to more than 10 miRNAs, 8582 to less than 10 miRNAs, and 2478 to a single miRNA.

There were 6 target genes that corresponded to $20 \mathrm{miR}$ NAs (Table 5). Methyl CpG binding protein 2 (MECP2) had the highest number of corresponding target genes, Sus scrofa glycogen synthase 1 (muscle) (GYS1), leucine rich repeat containing 8 family, member A (LRRC8A), B-cell CLL/lymphoma 9-like (BCL9L), PHD and ring finger domains 1 (PHRF1), ELOVL fatty acid elongase 1 (ELOVL1). Among 37 miRNAs, 24 miRNAs are thought to regulate MECP2, which may play a role in transcriptional regulation of neurons by directly regulating DGCR8/Drosha compounds and consequently influence the production of miRNA and expression of target genes (Chen, 2010; Wang et al., 2014; Woo and Kim, 2014).

Exosome signalling pathways analysis. Using KEGG analysis with the target prediction for 37 upregulated miRNAs in exosome, 9262 target genes were obtained, mainly concentrated on three signalling pathways, i.e. metabolic, cancer, and PI3K-Akt signalling pathways (Fig. 4). Three signalling pathways maintained a relationship with immunity and growth.

\section{Discussion}

We selected upregulated miRNAs in milk, performed basic sequencing, and examined the differential expression profile of miRNAs between exosomes and swine milk. We found that some miRNAs were selectively over-enriched by exosomes. Swine milk exosomes have been previously found to affect immunity by increasing the concentration of miRNAs ( $\mathrm{Gu}$ et al., 2012). We found six upregulated miRNAs: ssc-miR-423-5p, miR-193a, ssc-miR-551a, ssc-miR138, ssc-miR-1, and ssc-miR-124a. Urinary exosomal miR193a has been used as a biomarker for the diagnosis of primary focal segmental glomerulosclerosis in children (Huang et al., 2017; Khoo et al., 2017). Moreover, miR-193a-3p has been associated with tumour proliferation, transportation, and apoptosis (Yi et al., 2016). Five additional exosomal miRNAs, ssc-miR-423-5p, ssc-miR-551a, ssc-miR-138, ssc-miR-1, and ssc-miR-124a, were found to be concen- 
Table 3. Family analysis of upregulated miRNAs in exosomes.

\begin{tabular}{|c|c|c|c|}
\hline Number & Mature microRNA & Pre-miRNAs & Family \\
\hline 1 & ssc-miR-2 & ssc-mir-1 & $\operatorname{mir}-1$ \\
\hline 2 & ssc-miR-124a & ssc-mir-124a-2 & $\operatorname{mir}-124$ \\
\hline 3 & ssc-miR-124a & ssc-mir-124a-1 & $\operatorname{mir}-124$ \\
\hline 4 & ssc-miR-1272 & ssc-mir-1271 & $\operatorname{mir}-1271$ \\
\hline 5 & ssc-miR-130a & ssc-mir-129a & mir-129 \\
\hline 6 & ssc-miR-139 & ssc-mir-138 & mir-138 \\
\hline 7 & ssc-miR-140-4p & ssc-mir-140 & $\operatorname{mir}-140$ \\
\hline 8 & ssc-miR-146a-6p & ssc-mir-146a & mir-146 \\
\hline 9 & ssc-miR-147b & ssc-mir-146b & mir-146 \\
\hline 10 & ssc-miR-107a & ssc-mir-106a & $\operatorname{mir}-17$ \\
\hline 11 & ssc-miR-17-4p & ssc-mir-17 & $\operatorname{mir}-17$ \\
\hline 12 & ssc-miR-181d-6p & ssc-mir-181d & $\operatorname{mir}-181$ \\
\hline 13 & ssc-miR-186 & ssc-mir-185 & mir-185 \\
\hline 14 & ssc-miR-193a-4p & ssc-mir-193a & mir-193 \\
\hline 15 & ssc-miR-205 & ssc-mir-205 & mir-205 \\
\hline 16 & ssc-miR-210 & ssc-mir-210 & $\operatorname{mir}-210$ \\
\hline 17 & ssc-miR-219 & ssc-mir-219 & $\operatorname{mir}-219$ \\
\hline 18 & ssc-miR-221-4p & ssc-mir-221 & $\operatorname{mir}-221$ \\
\hline 19 & ssc-miR-223 & ssc-mir-222 & $\operatorname{mir}-221$ \\
\hline 20 & ssc-miR-92b-6p & ssc-mir-92b & $\operatorname{mir}-25$ \\
\hline 21 & ssc-miR-28a & ssc-mir-27a & $\operatorname{mir}-27$ \\
\hline 22 & ssc-miR-296-4p & ssc-mir-296 & mir-296 \\
\hline 23 & ssc-miR-30a-4p & ssc-mir-30a & $\operatorname{mir}-30$ \\
\hline 24 & ssc-miR-30c-4p & ssc-mir-30c-2 & $\operatorname{mir}-30$ \\
\hline 25 & ssc-miR-321 & ssc-mir-320 & mir-320 \\
\hline 26 & ssc-miR-331-4p & ssc-mir-331 & mir-331 \\
\hline 27 & ssc-miR-339-4p & ssc-mir-339-1 & mir-339 \\
\hline 28 & ssc-miR-34c & ssc-mir-34c-1 & $\operatorname{mir}-34$ \\
\hline 29 & ssc-miR-34c & ssc-mir-34c-2 & $\operatorname{mir}-34$ \\
\hline 30 & ssc-miR-345-3p & ssc-mir-345-1 & $\operatorname{mir}-345$ \\
\hline 31 & ssc-miR-345-3p & ssc-mir-345-2 & $\operatorname{mir}-345$ \\
\hline 32 & ssc-miR-365-6p & ssc-mir-365-2 & $\operatorname{mir}-365$ \\
\hline 33 & ssc-miR-378 & ssc-mir-378-1 & $\operatorname{mir}-378$ \\
\hline 34 & ssc-miR-378 & ssc-mir-378-2 & $\operatorname{mir}-378$ \\
\hline 35 & ssc-miR-423-6p & ssc-mir-423 & $\operatorname{mir}-423$ \\
\hline 36 & ssc-miR-455-3p & ssc-mir-455 & $\operatorname{mir}-455$ \\
\hline 37 & ssc-miR-455-5p & ssc-mir-455 & $\operatorname{mir}-455$ \\
\hline 38 & ssc-miR-552a & ssc-mir-551a & $\operatorname{mir}-551$ \\
\hline 39 & ssc-miR-4332 & ssc-mir-4331 & \\
\hline 40 & ssc-miR-4334-4p & ssc-mir-4334 & \\
\hline 41 & ssc-miR-7134-6p & ssc-mir-7134 & \\
\hline
\end{tabular}

Table 4. Statistics table of upregulated miRNA clusters in exosomes.

\begin{tabular}{lc|ll}
\hline Cluster & Number of pre-miRNA & \multicolumn{2}{|c}{ Pre-miRNAs } \\
\hline Cluster 1 & 2 & ssc-mir-34c-1 (9_44166873_44166952_-) & ssc-mir-34c-2 (9_44166877_44166950_-) \\
Cluster 2 & 2 & ssc-mir-221 (X_45274873_45274942_-) & ssc-mir-222 (X_45275613_45275692_-) \\
\hline
\end{tabular}

trated and upregulated (13.58 to 30.06-fold higher expression) in the exosomes, which are involved in the upregulation of important signalling molecules, such as p-AKT and p-ERK1/2. In clinical samples, miR-423-5p is dysregulated, and a corresponding alteration in ING-4 expression has been observed. Upregulation of miR-551a inhibits cell proliferation and induces apoptosis in demethoxycurcumin-hindered ovarian cancer (Du et al., 2017). miR-1 upregulates the expression of SFRP1 in bladder cancer cells; excessive miR-13 p's $3 p$ expression can stimulate the immune attack of blad- 
Table 5. Top 10 target genes corresponding to miRNAs.

\begin{tabular}{|c|c|c|c|c|}
\hline Targets & Gene & Description & $\begin{array}{l}\text { Number } \\
\text { of } \\
\text { miRNAs }\end{array}$ & miRNA \\
\hline $\begin{array}{l}\text { ENSSSCT } \\
00000035620\end{array}$ & MECP2 & $\begin{array}{l}\text { methyl CpG binding } \\
\text { protein } 2 \text { (Rett syn- } \\
\text { drome) }\end{array}$ & 24 & $\begin{array}{l}\text { ssc-miR-1271 | ssc-miR-138 | ssc-miR-140-3p | ssc-miR-146a-5p | } \\
\text { ssc-miR-17-3p | ssc-miR-185 | ssc-miR-205 | ssc-miR-219 | } \\
\text { ssc-miR-222 | ssc-miR-296-3p | ssc-miR-30c-3p | ssc-miR-320 | } \\
\text { ssc-miR-331-3p | ssc-miR-339-3p | ssc-miR-345-3p | ssc-miR-34c | } \\
\text { ssc-miR-365-5p | ssc-miR-378 | ssc-miR-423-5p | ssc-miR-4331 | } \\
\text { ssc-miR-4334-3p | ssc-miR-455-5p | ssc-miR-7134-5p | ssc-miR-92b-5p }\end{array}$ \\
\hline $\begin{array}{l}\text { ENSSSCT } \\
00000003502\end{array}$ & GYS1 & $\begin{array}{l}\text { Sus scrofa glycogen } \\
\text { synthase } 1 \text { (muscle) } \\
\text { (GYS1), mRNA }\end{array}$ & 21 & $\begin{array}{l}\text { ssc-miR-106a | ssc-miR-140-3p | ssc-miR-146a-5p | ssc-miR-17-3p | } \\
\text { ssc-miR-185 | ssc-miR-193a-3p | ssc-miR-210 | ssc-miR-296-3p | } \\
\text { ssc-miR-30c-3p | ssc-miR-320 | ssc-miR-331-3p | ssc-miR-339-3p | } \\
\text { ssc-miR-34c | ssc-miR-365-5p | ssc-miR-378 | ssc-miR-423-5p | } \\
\text { ssc-miR-4331 | ssc-miR-4334-3p | ssc-miR-551a | ssc-miR-7134-5p | } \\
\text { ssc-miR-92b-5p }\end{array}$ \\
\hline $\begin{array}{l}\text { ENSSSCT } \\
00000006227\end{array}$ & LRRC8A & $\begin{array}{l}\text { leucine rich repeat } \\
\text { containing } 8 \text { family, } \\
\text { member A }\end{array}$ & 20 & $\begin{array}{l}\text { ssc-miR-1271 | ssc-miR-138 | ssc-miR-140-3p | ssc-miR-146a-5p | } \\
\text { ssc-miR-185 | ssc-miR-193a-3p | ssc-miR-219 | ssc-miR-27a | } \\
\text { ssc-miR-30c-3p | ssc-miR-331-3p | ssc-miR-339-3p | ssc-miR-345-3p | } \\
\text { ssc-miR-34c | ssc-miR-365-5p | ssc-miR-378 | ssc-miR-4331 | } \\
\text { ssc-miR-4334-3p | ssc-miR-455-3p | ssc-miR-7134-5p | ssc-miR-92b-5p }\end{array}$ \\
\hline $\begin{array}{l}\text { ENSSSCT } \\
00000016465\end{array}$ & BCL9L & $\begin{array}{l}\text { B-cell CLL/lymphoma } \\
\text { 9-like }\end{array}$ & 20 & $\begin{array}{l}\text { ssc-miR-1271 | ssc-miR-138 | ssc-miR-17-3p | ssc-miR-185 | } \\
\text { ssc-miR-205 | ssc-miR-210 | ssc-miR-296-3p | ssc-miR-30a-3p | } \\
\text { ssc-miR-30c-3p | ssc-miR-320 | ssc-miR-331-3p | ssc-miR-339-3p | } \\
\text { ssc-miR-345-3p | ssc-miR-34c | ssc-miR-365-5p | ssc-miR-423-5p | } \\
\text { ssc-miR-4331 | ssc-miR-455-3p | ssc-miR-551a | ssc-miR-92b-5p }\end{array}$ \\
\hline $\begin{array}{l}\text { ENSSSCT } \\
00000024857\end{array}$ & PHRF1 & $\begin{array}{l}\text { PHD and ring finger } \\
\text { domains } 1\end{array}$ & 20 & $\begin{array}{l}\text { ssc-miR-1271 | ssc-miR-129a | ssc-miR-138 | ssc-miR-140-3p | } \\
\text { ssc-miR-181d-5p | ssc-miR-219 | ssc-miR-221-3p | ssc-miR-296-3p | } \\
\text { ssc-miR-30c-3p | ssc-miR-331-3p | ssc-miR-339-3p | ssc-miR-345-3p | } \\
\text { ssc-miR-365-5p | ssc-miR-378 | ssc-miR-423-5p | ssc-miR-4331 | } \\
\text { ssc-miR-4334-3p | ssc-miR-455-3p | ssc-miR-7134-5p | ssc-miR-92b-5p }\end{array}$ \\
\hline $\begin{array}{l}\text { ENSSSCT } \\
00000030861\end{array}$ & ELOVL1 & $\begin{array}{l}\text { ELOVL fatty acid } \\
\text { elongase } 1\end{array}$ & 20 & $\begin{array}{l}\text { ssc-miR-1 | ssc-miR-106a | ssc-miR-1271 | ssc-miR-140-3p | } \\
\text { ssc-miR-17-3p | ssc-miR-219 | ssc-miR-221-3p | ssc-miR-222 | } \\
\text { ssc-miR-27a | ssc-miR-296-3p | ssc-miR-30c-3p | ssc-miR-331-3p | } \\
\text { ssc-miR-339-3p | ssc-miR-345-3p | ssc-miR-34c | ssc-miR-378 | } \\
\text { ssc-miR-423-5p | ssc-miR-4331 | ssc-miR-455-5p | ssc-miR-92b-5p }\end{array}$ \\
\hline $\begin{array}{l}\text { ENSSSCT } \\
00000000472\end{array}$ & & $\begin{array}{l}\text { uncharacterized } \\
\text { protein }\end{array}$ & 19 & $\begin{array}{l}\text { ssc-miR-129a | ssc-miR-140-3p | ssc-miR-17-3p | ssc-miR-185 | } \\
\text { ssc-miR-205 | ssc-miR-219 | ssc-miR-221-3p | ssc-miR-222 | } \\
\text { ssc-miR-296-3p | ssc-miR-30c-3p | ssc-miR-331-3p | ssc-miR-339-3p | } \\
\text { ssc-miR-34c | ssc-miR-365-5p | ssc-miR-378 | ssc-miR-423-5p | } \\
\text { ssc-miR-4331 | ssc-miR-4334-3p | ssc-miR-7134-5p }\end{array}$ \\
\hline $\begin{array}{l}\text { ENSSSCT } \\
00000002656\end{array}$ & VASH1 & vasohibin 1 & 19 & $\begin{array}{l}\text { ssc-miR-1271 | ssc-miR-129a | ssc-miR-138 | ssc-miR-140-3p | } \\
\text { ssc-miR-193a-3p | ssc-miR-219 | ssc-miR-222 | ssc-miR-27a | } \\
\text { ssc-miR-296-3p | ssc-miR-30a-3p | ssc-miR-320 | ssc-miR-331-3p | } \\
\text { ssc-miR-339-3p | ssc-miR-34c | ssc-miR-365-5p | ssc-miR-4331 | } \\
\text { ssc-miR-4334-3p | ssc-miR-455-3p | ssc-miR-455-5p }\end{array}$ \\
\hline $\begin{array}{l}\text { ENSSSCT } \\
00000005027\end{array}$ & ZNF609 & zinc finger protein 609 & 19 & $\begin{array}{l}\text { ssc-miR-106a | ssc-miR-124a | ssc-miR-129a | ssc-miR-138 | } \\
\text { ssc-miR-140-3p | ssc-miR-146a-5p | ssc-miR-181d-5p | ssc-miR-185 | } \\
\text { ssc-miR-205 | ssc-miR-296-3p | ssc-miR-30c-3p | ssc-miR-320 | } \\
\text { ssc-miR-331-3p | ssc-miR-339-3p | ssc-miR-34c | ssc-miR-365-5p | } \\
\text { ssc-miR-378 | ssc-miR-423-5p | ssc-miR-92b-5p }\end{array}$ \\
\hline $\begin{array}{l}\text { ENSSSCT } \\
00000011944\end{array}$ & NAV1 & $\begin{array}{l}\text { uncharacterized } \\
\text { protein }\end{array}$ & 19 & $\begin{array}{l}\text { ssc-miR-106a | ssc-miR-1271 | ssc-miR-129a | ssc-miR-138 | } \\
\text { ssc-miR-140-3p | ssc-miR-17-3p | ssc-miR-185 | ssc-miR-296-3p | } \\
\text { ssc-miR-30c-3p | ssc-miR-320 | ssc-miR-331-3p | ssc-miR-34c | } \\
\text { ssc-miR-365-5p | ssc-miR-378 | ssc-miR-423-5p | ssc-miR-4331 } \\
\text { ssc-miR-4334-3p | ssc-miR-455-3p | ssc-miR-92b-5p }\end{array}$ \\
\hline
\end{tabular}




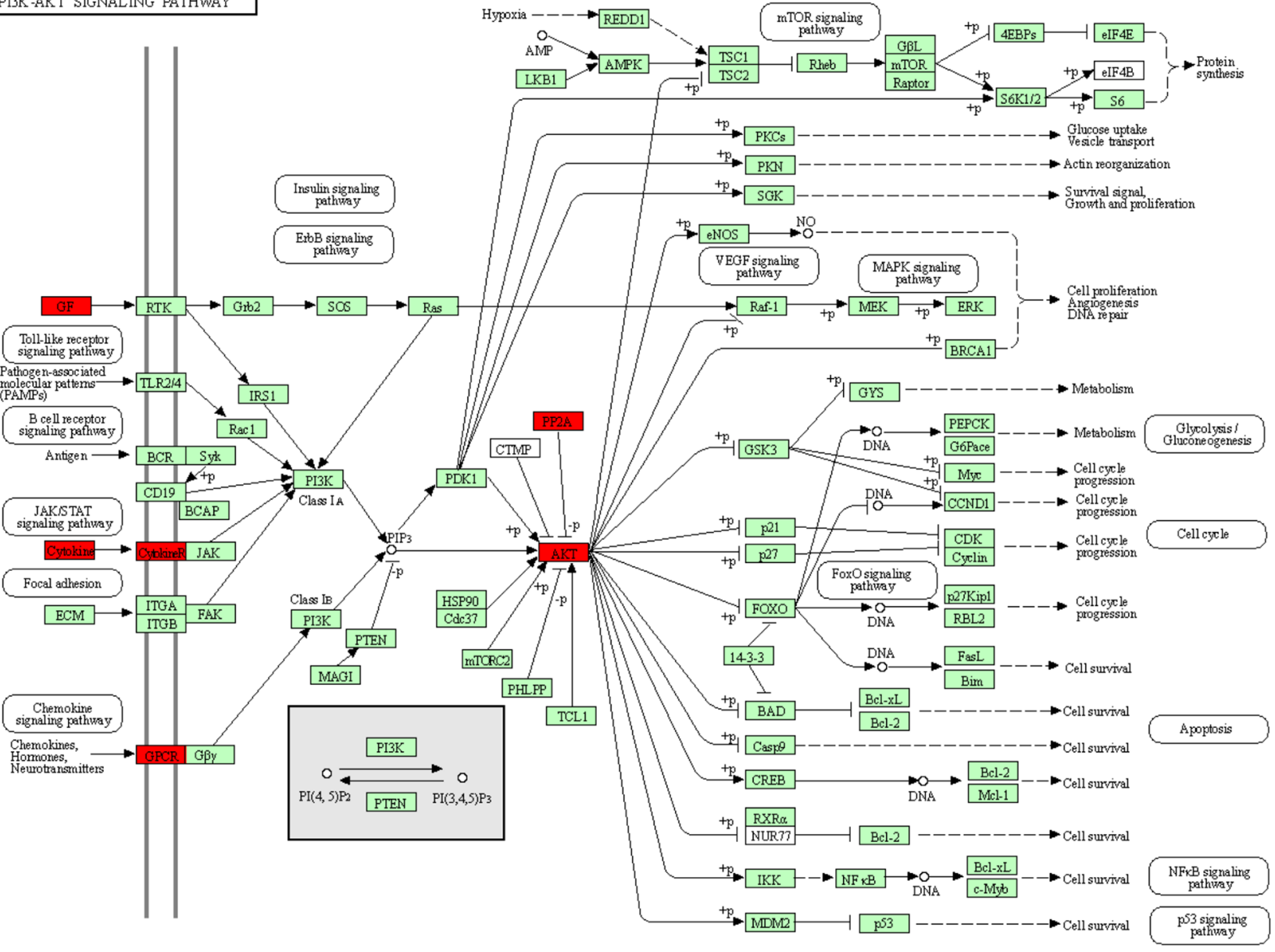

$041517 n / 21$
(c) Kanehisa Laboratories

Figure 4. The location of the upregulated miRNA target genes in the PI3K-Akt signalling pathway. The target genes of the upregulated miRNA are red.

der cancer cells, inhibiting their proliferation and movement (Shang et al., 2017). miR-124a can enhance SIRT1 and induce CD4 + T cells to differentiate Tregs, illustrating ways of influencing neuropathic pain inflammation (Heyn et al., 2016). In summary, these findings suggest that the miRNAs play a role in basic physiological processes of cell development and immunity.

There were 6 target genes that corresponded to $20 \mathrm{miR}$ NAs, MECP2, GYS1 BCL9L, PHRF1, and ELOVL1 (Table 5). Since most of the abundant miRNAs in exosomes can regulate $\mathrm{MECP} 2$, this may pave the way for the study of the effect of miRNAs in milk exosomes on the development of infant brains. Milk exosome miRNA-mediated suppression of DNMT1 and MECP2 may thus synergistically modify transcription and opening of chromatin structure, a critical epigenetic switch for growth and anabolism during the postnatal period (Melnik and Schmitz, 2017). GYS1 is interrelated with glycogen metabolism, and its mutation influences muscle glycogenosis (Baird et al., 2010; Fang et al., 2014).
LRRC8A gene codes are involved in different cell biological processes, including cell adhesion, transportation, and receptor-hormone interactions, and they play an important role in B cell development, whose defects result from B cell maturation deflection (Okada et al., 2016; Platt et al., 2017). BCL9L is an essential gene of growth and immunity. Lack of this gene can enhance chromosome endurance and regulate the proliferation of heteroploids through the Wnt signalling pathway (Roche et al., 2008; Sannino et al., 2016). PHRF1 affects the transportation into the nucleus of the cancer inhibitory factor cPML, blocking the signal transduction of TGF- $\beta /$ Smad. ELOVL1 has activity in fatty-acid-extended enzymes, impacting the metabolism of $\omega 3$ and $\omega 6$, which are related to some diseases, such as brain degeneration (LópezGarcía et al., 2017). Using chromosome localization and target gene analyses, we discovered that these genes were related to development, fat acid metabolism, and immunity. 
Furthermore, we found that the PI3K-Akt signalling pathway stimulated by various cells can regulate basic cellular functions, such as transcription, proliferation, translation, and development (Asati et al., 2016). PI3K is the second messenger that activates Akt, while PIP3 is catalysed by the cell membrane. Consequently, Akt could be relevant for functions such as apoptosis, generation of protein, metabolism, and cell cycle (Sizek et al., 2019). The finding that milkderived miRNAs that promote the PI3K-AKT pathway are abundantly expressed in exosomes supports the functional role of milk exosomal miRNAs in mammalian physiology, as previously suggested (Ettahar et al., 2013; Wang et al., 2018). Highly expressed miRNAs and their target genes participate in basic cell functions and processes such as development, metabolism, and immunity.

\section{Conclusions}

After performing in-depth research on miRNA localization, distribution, and function-related metabolism, we found that these exosome-concentrated miRNA target genes were interrelated with development and basic cell functions, such as metabolism and immunity. It is speculated that miRNAs in milk can influence offspring via milk exosomes.

Data availability. All data files are available from the SRA database (https://www.ncbi.nlm.nih.gov/sra) as of 1 October 2024, accession number: PRJNA760432.SRA, submission ID: SUB10315543.

Author contributions. ZL and YX made substantial contributions to the conception and design of the experiments. Conception and design of experiments was done by ZL, YX, and JL. Authors JG, ZL, YX, XS, CZ, and ChZ performed the experiments. Authors ZL and YX wrote the paper, and authors QQ, DD, YT, ZL, DW, and JL critically revised the manuscript. All authors read and approved the final manuscript.

Competing interests. The contact author has declared that neither they nor their co-authors have any competing interests.

Disclaimer. Publisher's note: Copernicus Publications remains neutral with regard to jurisdictional claims in published maps and institutional affiliations.

Acknowledgements. The authors are thankful the opinion of language errors from AJE company.

Financial support. This research has been supported by the National Natural Science Foundation of China (grant no. 32060742), the Major Science and Technology Projects of Inner Mongolia Autonomous Region (grant no. 2020ZD0004), the Key Technology Project of Inner Mongolia Autonomous Region (grant no. 2020GG0030) and the Youth fund of Double-first class project in Inner Mongolia Autonomous Region (grant nos. QN202102, QN202001 and QN201902).

Review statement. This paper was edited by Steffen Maak and reviewed by three anonymous referees.

\section{References}

Asati, V., Mahapatra, D. K., and Bharti, S. K.: PI3K/Akt/mTOR and Ras/Raf/MEK/ERK signaling pathways inhibitors as anticancer agents: Structural and pharmacological perspectives, Eur. J. Med. Chem., 109, 314-341, https://doi.org/10.1016/j.ejmech.2016.01.012, 2016.

Baird, J. D., Valberg, S. J., Anderson, S. M., Mccue, M. E., and Mickelson, J. R.: Presence of the glycogen synthase 1 (GYS1) mutation causing type 1 polysaccharide storage myopathy in continental European draught horse breeds, Vet. Rec., 167, 781784, https://doi.org/10.1136/vr.c3447, 2010.

Chen, X., Gao, C., Li, H., Huang, L., Sun, Q., Dong, Y., Tian, C., Gao, S., Dong, H., Guan, D., Hu, X., Zhao, S., Li, L., Zhu, L., Yan, Q., Zhang, J., Zen, K., and Zhang, C. Y.: Identification and characterization of microRNAs in raw milk during different periods of lactation, commercial fluid, and powdered milk products, Cell. Res., 20, 1128-1137, https://doi.org/10.1038/cr.2010.80, 2010.

Ettahar, A., Ferrigno, O. Zhang, M. Z., Ohnishi, N., Ferrand, N., Prunier, C., Levy, L., Bourgeade, M. F., Bieche, I., and Romero, D.: Identification of PHRF1 as a Tumor Suppressor that Promotes the TGF- $\beta$ Cytostatic Program through Selective Release of TGIF-Driven PML Inactivation, Cell. Rep., 4, 530-541, https://doi.org/10.1016/j.celrep.2013.07.009, 2013.

Fang, L., Shen, B., Irwin, D. M., and Zhang, S.: Parallel Evolution of the Glycogen Synthase 1 (Muscle) Gene Gys1 Between Old World and New World Fruit Bats (Order: Chiroptera), Biochem. Genet., 52, 443-458, https://doi.org/10.1007/s10528-014-96594, 2014.

Fu, F., Jiang, W., Zhou, L., and Chen, Z.: Circulating exosomal miR-17-5p and miR-92a-3p predict pathologic stage and grade of colorectal cancer, Transl. Oncol., 11, 221-32, doi.org/10.1016/j.tranon.2017.12.012, 2018.

Golan-Gerstl, R., Elbaum Shiff, Y., Moshayoff, V., Schecter, D., Leshkowitz D., and Reif, S.: Characterization and biological function of milk-derived miRNAs, Mol. Nutr. Food Res., 61, 10, https://doi.org/10.1002/mnfr.201700009, 2017.

Greening, D. W., Gopal, S. K., Xu, R., Simpson, R. J., and Chen, W.: Exosomes and their roles in immune regulation and cancer, Semin. Cell. Dev. Biol., 40, 72-81, https://doi.org/10.1016/j.semcdb.2015.02.009, 2015.

Gu, Y., Li, M., Liang, Z., Li, X., and Lv, X.: LactationRelated microRNA Expression Profiles of Porcine Breast Milk Exosomes, PLOS One, 7, e43691, https://doi.org/10.1371/journal.pone.0043691, 2012. 
Heyn, J., Luchting, B., Hinske, L. C., Hübner, M., Azad, S. C., and Kreth, S.: miR-124a and miR-155 enhance differentiation of regulatory $\mathrm{T}$ cells in patients with neuropathic pain, J. Neuroinflamm., 13, 248, https://doi.org/10.1186/s12974-016-07126, 2016.

Huang, Z., Zhang, Y., Zhou, J., and Zhang, Y.: Urinary Exosomal miR-193a Can Be a Potential Biomarker for the Diagnosis of Primary Focal Segmental Glomerulosclerosis in Children, Biomed. Res. Int., 29, 1-6, https://doi.org/10.1155/2017/7298160, 2017.

Khoo, C. P., Roubelakis, M. G., Schrader, J. B., Tsaknakis, G., Konietzny, R., Kessler, B., Harris, A. L., and Watt, S. M.: miR193a-3p interaction with HMGB1 downregulates human endothelial cell proliferation and migration, Sci. Rep., 7, 44137, https://doi.org/10.1038/srep44137, 2017.

Kosaka, N., Izumi, H., Sekine, K., and Ochiya, T.: microRNA as a new immune-regulatory agent in breast milk, Silence, 1, 7, https://doi.org/10.1186/1758-907X-1-7, 2010.

Lin, D. L., Chen, T., Meng, L. I., Xie, M. Y., Qian-Yun, X. I., Sun, J. J., and Zhang, Y. L.: Research Progress of miRNA in Milk, China J. Anim. Vet. Sci., 47, 1739-1748, available at: http://en. cnki.com.cn/Article_en/CJFDTotal-XMSY201609001.htm (last access: 20 January 2022), 2016.

Liu, Y., Wang, H., and Wang, J.: Exosomes as a novel pathway for regulating development and diseases of the skin, Biomed. Rep., 8, 207-214, https://doi.org/10.3892/br.2018.1054, 2018.

López-García, C., Sansregret, L., Domingo, E., Mcgranahan, N., Hobor, S., Birkbak, N. J., Horswell, E., Grönroos, E., Favaro, F., and Rowan, A. J.: BCL9L Dysfunction Impairs Caspase-2 Expression Permitting Aneuploidy Tolerance in Colorectal Cancer, Cancer Cell, 31, 7-93, https://doi.org/10.1016/j.ccell.2016.11.001, 2017.

Manca, S., Upadhyaya, B., Mutai, E., Desaulniers, A. T., Cederberg, R. A., White, B. R., and Zempleni, J.: Milk exosomes are bioavailable and distinct microRNA cargos have unique tissue distribution patterns, Sci. Rep., 8, 11321, https://doi.org/10.1038/s41598-018-29780-1, 2018.

Melnik, B. C. and Schmitz, G.: Milk's Role as an Epigenetic Regulator in Health and Disease, Diseases, 5, 12, https://doi.org/10.3390/diseases5010012, 2017.

Melnik, B. C., Kakulas, F., Geddes, D. T., Hartmann, P. E., John, S. M., Carrera-Bastos, P., Cordain, L., and Schmitz, G.: Milk miRNAs: simple nutrients or systemic functional regulators, Nutr. Metab., 13, 42, https://doi.org/10.1186/s12986-016-0101$2,2016$.
Okada, T., Islam, M. R., Tsiferova, N. A., Okada, Y., and Sabirov, R. Z.: Specific and essential but not sufficient roles of LRRC8A in the activity of volume-sensitive outwardly rectifying anion channel (VSOR), Channels, 11, 109-120, https://doi.org/10.1080/19336950.2016.1247133, 2016.

Platt, C. D., Chou, J., Houlihan, P., Badran, Y. R., Kumar, L., Bainter, W., Pliani, P. L., Perez, C. J., Dent, S. Y., and Clapham, D. E.: Leucine-rich repeat containing 8A (LRRC8A)-dependent volume-regulated anion channel activity is dispensable for T-cell development and function, J. Allergy Clin. Immun., 140, 16511659, https://doi.org/10.1016/j.jaci.2016.12.974, 2017.

Roche, M. D. L., Worm, J., and Bienz, M.: The function of BCL9 in $\mathrm{Wnt} / \beta$-catenin signaling and colorectal cancer cells, BMC Cancer, 8, 199, https://doi.org/10.1186/1471-2407-8-199, 2008.

Sannino, G., Armbrster, N., Bodenhöfer, M., Haerle, U., Behrens, D., Buchholz, M., Rothbauer, U., Sipos, B., and Schmees, C.: Role of BCL9L in transforming growth factor- $\beta$ (TGF$\beta$ )-induced epithelial-to-mesenchymal-transition (EMT) and metastasis of pancreatic cancer, Oncotarget, 7, 73725-73738, https://doi.org/10.18632/oncotarget.12455, 2016.

Shang, A., Yang, M., Shen, F., Wang, J., Wei, J., Wang, W., Lu, W., Wang, C., and Wang, C.: MiR-1-3p Suppresses the Proliferation, Invasion and Migration of Bladder Cancer Cells by Up-Regulating SFRP1 Expression, Cell. Physiol. Biochem., 41, 1179-1188, https://doi.org/10.1159/000464379, 2017.

Sizek, H., Hamel, A., Deritei, D., Campbell, S., and Ravasz Regan, E.: Boolean model of growth signaling, cell cycle and apoptosis predicts the molecular mechanism of aberrant cell cycle progression driven by hyperactive PI3K, PLOS Comput. Biol., 15, e1006402, https://doi.org/10.1371/journal.pcbi.1006402, 2019.

Wang, L., Sadri, M., Giraud, D., and Zempleni, J.: RNase $\mathrm{H}_{2}$-Dependent Polymerase Chain Reaction and Elimination of Confounders in Sample Collection, Storage, and Analysis Strengthen Evidence That microRNAs in Bovine Milk Are Bioavailable in Humans, J. Nutr., 148, 153-159, https://doi.org/10.1093/jn/nxx024, 2018.

Yi, Y., Chen, J., Jiao, C., Zhong, J., Song, Z., Yu, X., Lu, X., and Lin, B.: Upregulated miR-193a-3p as an oncogene in esophageal squamous cell carcinoma regulating cellular proliferation, migration and apoptosis, Oncol. Lett., 12, 4779-4784, https://doi.org/10.3892/ol.2016.5229, 2016.

Zhang, M., Zhang, C. G., and Ding, W.: Exosome in Cancer Diagnosis and Treatment, Prog. Physiol. Sci., 45, 372-378, 2014. 